\title{
Teaching Basic Quantum Mechanics in Secondary School Using Concepts of Feynman Path Integrals Method
}

María de los Ángeles Fanaro and María Rita Otero, Universidad Nacional del Centro de la Provincia de Buenos Aires, Tandil, Argentina

Marcello Arlego, Universidad Nacional de La Plata, La Plata, Argentina

$\mathrm{T}$ his paper discusses the teaching of basic quantum mechanics in high school. Rather than following the usual formalism, our approach is based on Feynman's path integral method. Our presentation makes use of simulation software and avoids sophisticated mathematical formalism.

We start by discussing the experimental results that would be observed in an idealized double-slit experiment. With the assistance of software simulations, we focus on the changes observed as the objects' masses are reduced from macroscopic to atomic values. After that we introduce the basis of quantum mechanics by means of the concepts of path integrals, which henceforth we will call "sum of all alternatives." Finally, we show how the transition from classical to quantum behavior naturally emerges within the framework of "sum of all alternatives."

\section{The instructional sequence}

The material was used in a senior-level high school physics course. The class was composed of 30 students who were 1718 years old. There were two one-hour class periods per week. The students had already been exposed to trigonometric functions, vectors, and classical mechanics. The instructional sequence consisted of 13 lessons divided into four stages as follows:

\section{Double-slit experience with small balls and electrons}

Our students imagined and predicted the results of this experience using small balls. Then, the experiments were simulated using the software Doppelspaltversuch. ${ }^{1}$ The students compared their predictions about the distribution of particles on the screen with the results of the simulation and concluded that: "When both slits are open, the resulting distribution curve is the sum of the individual curves."

After that the students analyzed the double-slit experiment with electrons instead of small balls. The simulation showed that electrons distributed on the screen very differently than small balls did. The result was inexplicable from the classical theory and so the idea that electrons would be as small balls was found to be naive.

Although some students could not identify the distribution with the interference pattern observed in experiments with mechanical waves (for instance), they accepted another key principle: "When both slits are open, the distribution of electrons on the screen does not follow a pattern that can be produced by the separate contributions of particles emerging from each slit."

This convinced the students that it is inadequate to consider electrons as particles, at least in a classical sense. This new way of considering the electrons drove us to introduce the concept of "quantum system."

\section{Analysis and application of the "sum of all alter- natives" method for free electrons}

We started by stating that there exists a set of laws that describe the behavior of objects ranging from macroscopic to atomic scale. They are called the laws of quantum mechanics. These predict only the probability of an event. That is to say, given an initial state, what is the probability of arriving at a particular final state? We adopted the Feynman formulation of quantum mechanics and adapted it to our students' mathematic level. We replaced complex numbers by twodimensional vectors, integrals by finite sums, and derivatives by finite ratios.

The "sum of all alternatives" formulation of quantum mechanics consists of the following steps: ${ }^{2}$

1. - Suppose a particle's initial state $(I)$ is defined by its location at time $=0, x(t=0)=0$. In its final state $(F)$, at time $=T$, $x(t=T)=x_{\mathrm{f}}$. See Fig. 1 . We consider here one-dimensional paths for simplicity.

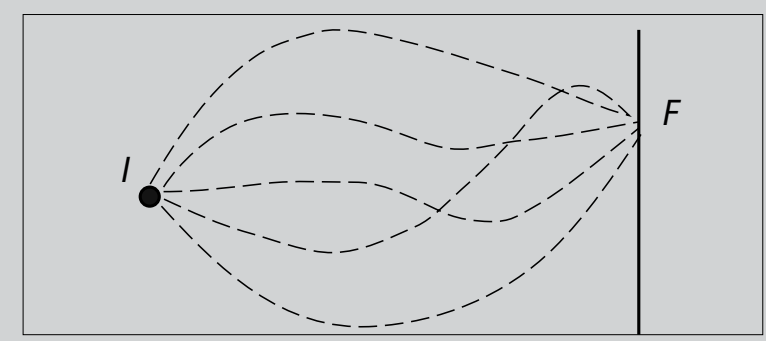

Fig. 1. Possible paths to connect I with $F$.

2. -With each possible path $x(t)$ (some of them are shown in Fig. 1) we associate a numerical value called "action," represented by $S$. The action is the average difference between the kinetic energy $E_{k}$ and potential energy $E_{p}$ multiplied by $T$ : 


$$
S=<E_{\mathrm{k}}-E_{\mathrm{p}}>\cdot T,
$$

where $<>$ denotes the average over time. If the particle is "free" $\left(E_{\mathrm{p}}=0\right)$, the action is simply $\mathrm{S}=\left\langle E_{\mathrm{k}}>\cdot T\right.$, i.e ,

$$
S=1 / 2 m\left\langle v^{2}\right\rangle \cdot T \text {. }
$$

Every path $x(t)$ connecting $I$ with $F$ has a corresponding $S$, which is used to construct the amplitude of probability vector associated with $x(t):[\cos (s / \hbar), \sin (s / \hbar)]$,

where $\hbar=h / 2 \pi$ and $h$ is Planck's constant.

3. - When all the probability amplitude vectors associated with all possible paths connecting $I$ with $F$ are added, we call the resulting vector the "total probability amplitude" $\left[A_{t}(x)\right]$.

4. - The square modulus of the total probability amplitude gives the relative probability $P$ of arriving at final state $F$, having started at initial state $I$, i.e., $P \propto\left|A_{\mathrm{t}}(x)\right|^{2}$

To help the students to apply the technique of "sum of all alternatives" to the free electron, a simulation using Modellus ${ }^{3}$ was developed. The use of this simulation software enabled the evaluation of the contributions of different paths to the probability amplitude. It allowed students to formulate the following conclusions:

i) The classical path $x_{\text {class }}(t)$ (a straight line from $I$ to $F$ ) has the least action $S$.

ii) For atomic masses, the angles of the amplitude vectors associated with those paths $x(t)$ near the classical path $x_{\text {class }}(t)$ are very similar. However, the angles of the vectors associated with paths $x(t)$, which are far from the classical path, are different from each other. Therefore, only a set of paths "around" the classical path contributes to the sum. The others have very different directions and cancel

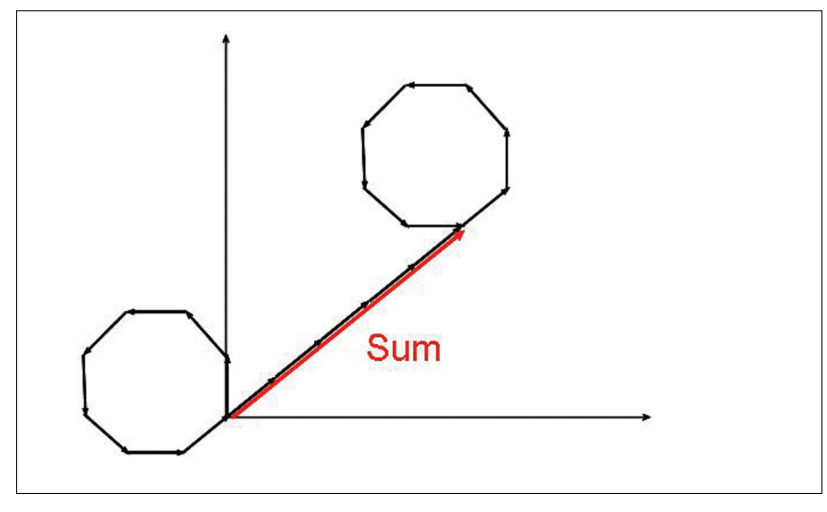

Fig. 2. Schematic representation of the sum of amplitudes for a finite set of trajectories connecting initial and final states. Every vector corresponds to every function $x(t)$ connecting $I$ with $F$. All of them are unit vectors and form an angle $S / \hbar$ with the positive $x$-axis. Some vectors were selected, and the sum was done using the tail-to-head method. Only trajectories near the classical one contribute coherently to the sum (red straight line). See text for more details. each other in the sum. It was emphasized that this is so for the free electron. In general in a quantum system all paths contribute to the sum. Figure 2 is a schematic representation of the sum for $V=0$.

iii) As the particle masses increase, the contributions to the total amplitude are reduced to paths extremely close to the classical path $x_{\text {class }}(t)$. In the limiting case of a macroscopic object, only the classical path contributes to the sum. The mechanism of cancellation (Fig. 2), independently of $V$, is due to the very large values of $S$ (with respect to $\hbar$ ). Therefore, in a macroscopic context, quantum mechanics predicts the same results as classical physics, i.e., there is only one trajectory, the one of least action.

iv) It is the quotient $S / \hbar$ which determines the classical $(S>>$ $\hbar)$ or quantum $(S \approx \hbar)$ character of a system.

\section{Applying the "sum of all alternatives" method to reconstruct the interference diagram with elec- trons}

In the double-slit experiment, the probability for a particle of mass $m$ to arrive at a distance $x$ from second screen center, starting from the source, yields the following result (see online appendix for a derivation adapted to students' mathematical level ${ }^{4}$ ).

$$
P(x) \propto 4 \cos ^{2}\left(\frac{m d}{2 \hbar T} x\right),
$$

where $d$ is the distance between slits on the first screen. The students discussed and analyzed in groups the functional form of the expression $P(x)$ given above. They recognized that $P(x)$ represents the distribution of electrons obtained in the simulations.

\section{Classical-quantum transition in the double-slit experiment}

In order to show how the shape of the curve $P(x)$ changes with the mass, an additional Modellus simulation was generated. Students observed that the distance between maxima and minima was reduced as the mass is increased, while keeping all other parameters fixed.

Figure 3 illustrates this behavior, showing how the interference diagram disappears as the mass increases, making evident the transition between quantum and classical mechanics.

From previous observations students identified a wavelength to be dependent on the ratio $\hbar / m v$, where $v \approx R_{1,2} / T$ (where $R_{1,2}$ is the average distance from each slit to $x$ ). It depends only on the properties of the particle, so it makes sense to associate this wavelength with the particle itself. In this way we arrive at the concept of wavelength $\lambda$ associated with the particle. This is called "de Broglie wavelength" and it is given by

$$
\lambda \approx \frac{\hbar}{p},
$$

where $p=m v$ is the classical momentum of the particle. 


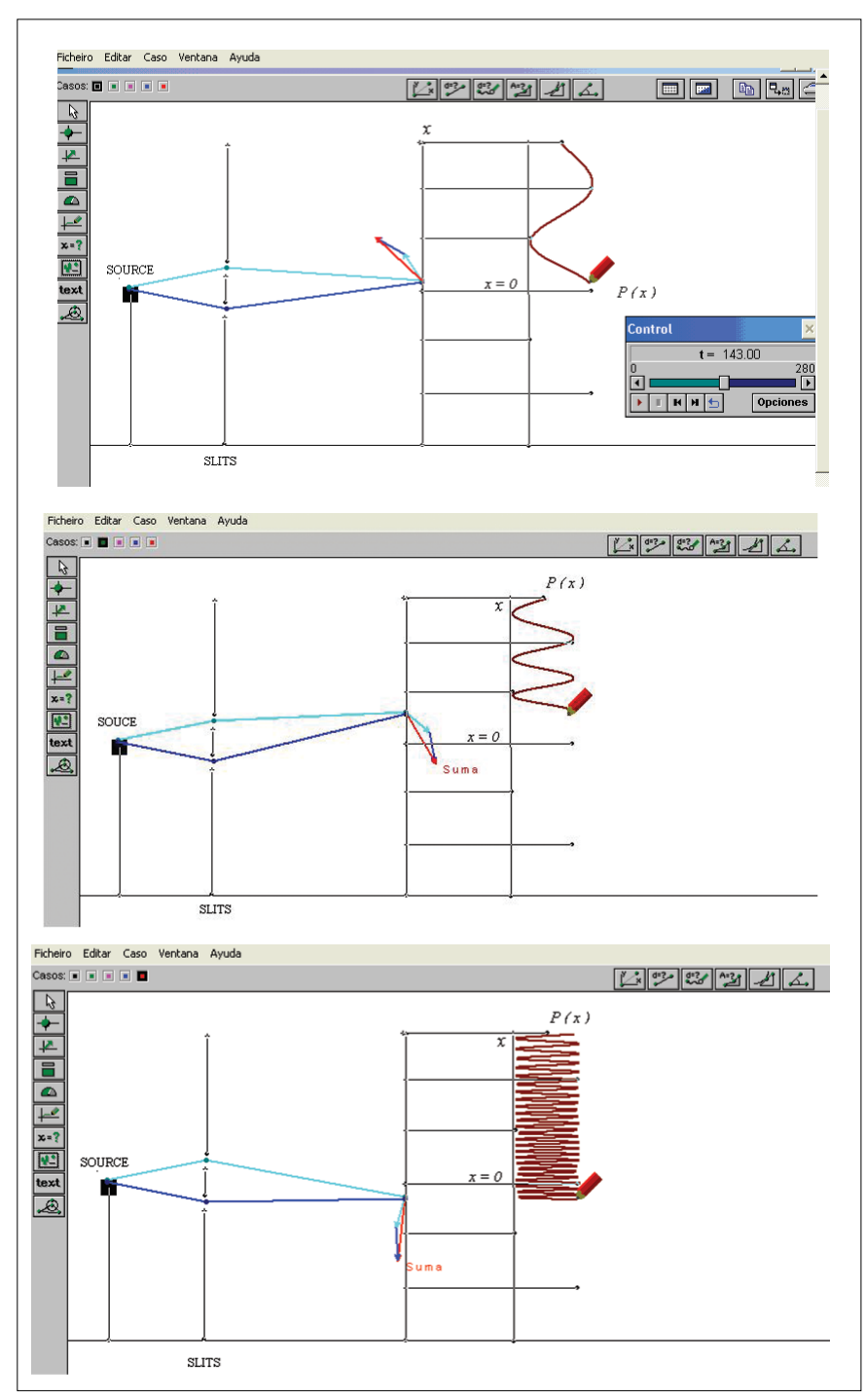

Fig. 3. Transition between quantum (top) to classical behavior (bottom) in the double-slit experiment. The screens show the simulation of the double-slit exercise using Modellus. Observe how the distances between maxima and minima are reduced, making the interference pattern disappear, as the mass is increased (top, middle, and bottom panels, respectively).

From this formula it is clear that it is the ratio between $p$ and $h$ that determines the undulatory character of the system. For macroscopic objects $\lambda$ is so small that undulatory characteristics are imperceptible. On the other hand for electrons, for instance, $\lambda$ can be large enough to exhibit interference effects.

Finally, let us note that in terms of the DeBroglie wavelength, the cosine argument in the probability distribution has the form $2 \pi \Delta R / \lambda$, with $\Delta R=\left|R_{1}-R_{2}\right|$ (see appendix). ${ }^{4}$

The sequence ended showing photographs of a real double-slit experience with electrons, ${ }^{5}$ showing how the interference pattern on the screen is made up from the individual impacts of electrons.

\section{Conclusions}

We have developed an alternative method for teaching the fundamentals of QM for secondary school students based on Feynman's path integrals. Our work has focused on the emergence of quantum behavior in idealized double-slit experiments employing material objects with masses ranging from macroscopic to atomic levels (e.g., electrons).

The results were satisfactory; students expressed on many occasions that they had made an intense but successful effort. They were not overwhelmed by the proposed situations and they accepted the challenges. The results of the final test were satisfactory. 6,7

Regarding software aspects, students also recognized their advantages to support the understanding of new concepts. As a continuation of the topics discussed here, we plan to address the quantum aspects of light, in particular the emergence of the concept of the photon, in a future work.

\section{Acknowledgments}

CONICET Consejo Nacional de Investigaciones Científicas y Técnicas. Ministerio de Ciencia, Tecnología e Innovación Productiva. Presidencia de la Nación - República Argentina, www.conicet.gov.ar/.

\section{References}

1. Doppelspalversuch (2003). By K. Muthsam (Version 3.3, translated to Spanish by Wolfamann y Brickmann), Physics Education Research Group of the University of Munich. It can be found on the web: homepages.physik.uni-muenchen.de/ milq/ download.html.

2. It is important to stress that steps 1-4 are an adaptation of the laws of quantum mechanics to secondary school. These laws are fundamental in the sense that they cannot be derived from more basic principles. On the other hand these laws predict results that are in agreement with the experiment, which validates the theory. For instance, it correctly predicts the distribution of electrons in the double-slit experiment.

3. Modellus ${ }^{\mathrm{TM}}$ version 2.5, developed by Victor Duarte Teodoro and Joao Paulo Duque Viera, Filipe Costa Clérigo Faculty of Sciences and Technology Nova University, Lisbon, Portugal. The simulations created are available at mariaangelesfanaro. sites.exa.unicen.edu.ar/simulaciones-con-modellus.

4. Online appendix can be viewed at dx.doi.org.10.1119/1.3685112.

5. Images obtained from en.wikipedia.org/wiki/Double-slit_ experiment.

6. M. Fanaro and M. R. Otero, "Basics quantum mechanics teaching in secondary school: One conceptual structure based on paths integrals method," Lat. Am. J. Phys. Educ. 2 (2), 103-112 (2008).

7. M. Fanaro, M. R. Otero, and M. A. Arlego, "Teaching the foundations of quantum mechanics in secondary school: A proposed conceptual structure," in Investigações em Ensino de Ciências 14(1), 37-64 (2009). 